\title{
The Generalized Reaction Rate Theories in the Systems with Power-Law Distributions
}

\author{
Cangtao Yin
}

The calculations of reaction rate are important in studying the different processes in physics, chemistry, biology, engineering etc. There exist many theories of reaction rate, such as transition state theory, collision theory, and unimolecular reaction theory. One key assumption in these theories is that thermodynamic equilibrium prevails throughout the entire system studied for all degrees of freedom. According to the Boltzmann-Gibbs statistical mechanics, a Maxwell-Boltzmann distribution whose form is exponential law holds in the whole time. This exponential law form of reaction rate is usually called Arrhenius behavior in chemistry. However, plenty of experimental results show non-Arrhenius behavior, such as power-law behavior. Besides, in reacting systems, the processes of evolution from one meta-stable state to another neighboring metastable state happen all the time, therefore the equilibrium assumption would be quite far-fetched. In these cases, current theories are no longer valid, and they can not even serve as a conceptual guide for understanding the critical factors that determine rates. Therefore, providing corresponding theoretical description for non-Arrhenius behavior or power-law behavior becomes urgent. In this general paper, generalized reaction rate theories with power-law distributions in the framework of nonextensive statistical mechanics were discussed based on experimental and observing facts.

Keywords: reaction rate theory, power-law distribution, non-Arrhenius, nonextensive statistics 


\section{Introdução}

The power-law behavior in nonequilibrium systems have been found and noted prevalently in the processes such as anomalous diffusion in anomalous media [1, 2], dissipative biological systems [3, 4], folding of proteins [5], combustion processes [6], gene expressions [7], small organic molecules [8], cell reproductions [9], complex cellular networks [10] etc, which are beyond the scope of conventional reaction rate theory with Maxwell-Boltzmann distributions in equilibrium systems. In these cases, the traditional rate formulae of reaction rate theory become invalid and so need to be modified. In recent years, a new kind of statistical mechanical theory -- Nonextensive Statistical Mechanics has been constructed and received great attention, which is based on the q-entropy proposed by Tsallis [11], and has very wide applications for a variety of interesting problems on systems beyond equilibrium [12]. It has become a very useful tool to approach nonequilibrium systems whose properties go beyond the realm governed by Boltzmann-Gibbs statistical mechanics. nonextensive statistical mechanics now naturally gives rise to a possibility for us to generalize the conventional reaction rate theory to nonequilibrium systems presenting the non-Arrhenius behavior, which is just the purpose in this paper.

There existing various reaction rate theories that have been developed to calculate the reaction rate. In all the traditional theories, the distribution function has always been assumed to be a Maxwell-Boltzmann distribution. This is only a good approximation in the situations when the statistical property of the system can be described by Boltzmann-Gibbs statistical mechanics. However, chemical reaction systems are generally far from thermal equilibrium and therefore the statistical properties do not exactly obey MaxwellBoltzmann distribution. Based on nonextensive statistical mechanics, three kinds of reaction rate theories to nonequilibrium system presenting the nonArrhenius behavior have been generalized, i.e., the generalized transition state theory, the generalized collision theory, and the generalized unimolecular reaction theory.

\section{Fundamentals}

\section{GENERALIZED TRANSITION STATE THEORY}

Among various reaction rate theories that have been developed to calculate the reaction rate, transition state theory is the most basic one [13]. Transition state theory has made it possible to obtain quick estimates for the reaction rates of a broad variety of processes in natural science and thus became a cornerstone and a core of the reaction rate theory. By generalizing the equilibrium constant and the chemical potential in the framework of nonextensive statistical mechanics, the generalized transition state theory reaction rate formula for the elementary reaction (A $+\mathrm{BC} \rightarrow \mathrm{A} \cdots \mathrm{B} \cdots \mathrm{C} \rightarrow \mathrm{AB}+\mathrm{C}$ ) taking place in the system presenting non-Arrhenius behavior was obtained under the assumption of one dimensional reaction coordinate motion [14],

$$
k_{q-\mathrm{TST}}=\omega_{f}\left[2+\left(\frac{k_{B} T}{h \omega_{f}}\right)^{1-q}\left(Z_{\Delta}^{\neq}\right)^{1-q}-Z_{A}^{1-q}-Z_{B C}^{1-q}-(1-q) \frac{\Delta \varepsilon_{0}}{k_{B} T}\right]^{\frac{1}{1-q}},
$$

where $k$ is the reaction rate coefficient. $q$ represents the statistical property of a system being at nonequilibrium stationary-state $[11,12]$. TST stands for transition state theory. The system is reduced to a Maxwell-Boltzmann distribution if the q-parameter is set $\mathrm{q} \rightarrow 1$, then the parameter $\mathrm{q} \neq 1$ measures a distance away from the equilibrium and it is a function of the energy. $\omega_{f}$ is the reaction coordinate frequency (i.e. decomposition frequency of transition state), then $1 / \omega_{f}$ is the mean lifetime of the transition state. $\mathrm{h}$ is Planck constant. $k_{B}$ is Boltzmann constant and $T$ is temperature. $Z$ is a partition function. $\neq$ denotes the transition state. The reaction coordinate motion can be separated from the whole motion in the transition state, therefore the whole partition function $Z^{\ddagger}$ for the transition state can be written as $Z^{1}=Z^{1}{ }_{w} Z_{D}^{1}, Z^{1}$, is the transition state partition function which removed the vibration partition function $Z_{{ }_{w}}^{1} . \Delta \varepsilon_{0}$ is the difference of basic energies between transition state and reactants.

As expected, in the limit $q \rightarrow 1$, Eq.(1) is reduced to the standard transition state theory reaction rate formula for the elementary reaction in systems with a Maxwell- 
Boltzmann distribution $[15,16]$.

It was found that the power-law transition state theory reaction rate formula Eq. (1) not only depends on the $q$-parameter, but also on the reaction coordinate frequency $\omega_{f}$ which does not appear in the conventional transition state theory reaction rate formula.

In addition, the tunneling correction was considered into generalized transition state theory, which could be very important for the reactions in low temperature. The power-law transition state theory reaction rate formula with the tunneling correction could be expressed as [17],

$$
\begin{aligned}
& k_{q-T w n}=\kappa_{q} k_{q-T S T} \\
& =\frac{(2-q) \omega_{f}}{k_{B} T}\left[2+\left(\frac{k_{B} T}{h \omega_{f}} Z_{\Delta}^{z}\right)^{1-q}-\sum_{i} Z_{i}^{1-q}-\frac{(1-q) \Delta \varepsilon_{0}}{k_{B} T}\right]^{\frac{1}{1-q}}\left[1-\frac{(1-q) \Delta \varepsilon_{0}}{k_{B} T}\right]^{\frac{q-2}{1-q}}, \\
& \quad \times \int_{0}^{\infty} d \varepsilon \frac{\cosh \left(2 \alpha \zeta^{1 / 2}\right)-1}{\cosh \left(2 \alpha \zeta^{1 / 2}\right)-\cosh \left(\sqrt{4 \alpha^{2}-\pi^{2}}\right)}\left[1-\frac{(1-q) \varepsilon}{k_{B} T}\right]^{\frac{1}{1-q}}
\end{aligned}
$$

where $\zeta=\varepsilon / \Delta \varepsilon_{0}$ and $\alpha=2 \pi \Delta \varepsilon_{0} / \mathrm{h} \omega_{f}, k$ is tunneling correction with Eckart barrier [18]. $Z i$ is the partition function of the $i$ th reactant molecule. As expected, in the limit $\mathrm{q} \rightarrow 1$, Eq. (2) recovers the standard form of the transition state theory reaction rate formula with tunneling correction in Boltzmann-Gibbs statistics [18, 19].

\section{Generalized Collision Theory}

The collision theory is an old and foundational reaction rate theory among all the theories calculating the reaction rate [20]. More important, analysis of the collision phenomena plays a central role in almost all investigations of structures of matters on microscopic scale. As a first step of the generalization of the collision theory rate formula to the nonequilibrium system presenting non-Arrhenius behavior, a simple system involving two gases, A and B, whose molecules behave as hard spheres characterized by the impenetrable radii $R_{A}$ and $R_{B}$, was considered [21]. The collision between $\mathrm{A}$ and $\mathrm{B}$ occurs when their centers approach within a distance $d_{A B}$, such that $d_{A B}=R_{A}+R_{B}$. By using the nonextensive velocity distribution [22], the collision theory reaction rate for systems presenting non-Arrhenius behavior was derived, and the new collision theory can overcome the difficulties in the Lindemann-Christiansen mechanism. The collision theory reaction rate coefficient for the power-law distribution was derived by [21],
$k_{q-C T}=\chi_{q} \pi d_{A B}^{2} \sqrt{\frac{8 k_{B} T}{\pi \mu}}\left[1-(1-q) \frac{\varepsilon_{c}}{k_{B} T}\right]_{+}^{\frac{2-q}{1-q}}$,

with the $q$-dependent parameter,

$$
\chi_{q}=\left\{\begin{array}{c}
\Gamma[1 /(1-q)+5 / 2] /(1-q)^{1 / 2} \Gamma[1 /(1-q)+3], \quad q<1, \\
\Gamma[1 /(q-1)-2] /(q-1)^{1 / 2} \Gamma[1 /(q-1)-3 / 2], \quad 1<q<3 / 2 .
\end{array}\right.
$$

CT means collision theory. $\mu$ is reduced mass. $\varepsilon_{\mathrm{c}}$ is the critical energy value at which the reaction occurs. As expected, when taking the limit $q \rightarrow 1$, it become the familiar form in the conventional collision theory [20].

What's more, the reaction without barrier with powerlaw distribution was studied in the framework of Tsallis statistics in the Gorin model $[23,24]$. The reaction rate coefficient for the barrierless reaction in the system with power-law velocity q-distribution was derived as [25],

$$
k_{q-R N B}=2^{11 / 6} \Gamma(2 / 3) \sqrt{\pi / \mu} C^{1 / 3}\left(k_{B} T\right)^{1 / 6} K_{q},
$$

with the $q$-dependent factor,

$$
K_{q}=\left\{\begin{array}{c}
(1-q)^{-1 / 6} \Gamma[1 /(1-q)+5 / 2] / \Gamma[1 /(1-q)+8 / 3], \quad q<1, \\
(q-1)^{-1 / 6} \Gamma[1 /(q-1)-5 / 3] / \Gamma[1 /(q-1)-3 / 2], 1<q<8 / 5
\end{array}\right.
$$

RWB stands for reaction without barrier, $C$ is a constant determined by the nature of the molecules.

\section{Generalized Unimolecular Reaction Theory}

Unimolecular reactions are in principle the simplest 
type of chemical reactions that can occur in the gas phase since the reactions formally involve only one molecule. The unimolecular reaction theory in the gas phase is a classical topic in physical chemistry [15, 19]. The reaction rate of inimolecular reactions in high and low pressure limits should be studied respectively.

In high pressure limit, by generalizing the energy distribution function $\mathrm{P}(\mathrm{E})$, the generalized unimolecular reaction theory rate formula for the nonequilibrium system presenting power-law behavior was obtained [26],

$$
k_{q-v n i}^{\infty}=\frac{\prod_{i=1}^{s} v_{i}}{\prod_{i=1}^{s-1} v_{i}^{*}}\left[1-(1-q) \frac{E_{0}}{k_{B} T}\right]^{\frac{1}{1-q}+s},
$$

where $E_{0}$ is the threshold energy. If there are s degrees of freedom in the reactant with frequencies vi, there are $s^{-1}$ degrees of freedom in the transition state with frequencies $v_{i}^{\neq}$when the reaction coordinate is excluded.

In the same way, the unimolecular reaction theory rate in the low pressure limit was also generalized in the framework of nonextensive statistical mechanics [26],

$$
k_{q-\text { uni }}^{0}=\frac{Z_{A M}}{(s-1) !}\left\{\begin{array}{l}
B\left(\frac{(q-1) E_{0}}{k_{B} T+(q-1) E_{0}}, s, \frac{1}{q-1}-s\right) \Gamma\left(\frac{1}{q-1}\right) / \Gamma\left(\frac{1}{q-1}-s\right), 1<q<1+\frac{1}{s}, \\
B\left(\frac{(1-q) E_{0}}{k_{B} T}, s, \frac{1}{1-q}+1\right) \Gamma\left(\frac{1}{1-q}+1+s\right) / \Gamma\left(\frac{1}{1-q}+1\right), \quad q<1,
\end{array}\right.
$$

where $Z_{A M}$ is the collision number between reactant molecule $\mathrm{A}$ and $\mathrm{M}$ per unit volume per unit time. ( $\mathrm{M}$ is a insert/buffer gas, or any molecule that does not react with the molecule A, which could be A itself.) B is the upperpart incomplete Beta function [27].

It is clear that by taking the limit $q \rightarrow 1$ in both Eq. (5a) and (5b) the standard unimolecular reaction theory rate coefficient for the system with a Maxwell-Boltzmann distribution [28] can be recovered perfectly.

The new rate formulae depend strongly on the powerlaw parameter, in both the high and low pressure limits. the new unimolecular reaction theory rate formula depends on the number of degrees of freedom s, which does not appear in the traditional unimolecular reaction theory formula.

\section{Applications}

In order to illustrate the application of the generalized reaction rate theories above to chemical reactions occurring in a nonequilibrium system with the power-law distributions, several typical reactions as examples were taken to testify the generalized formulae.

For generalized transition state theory, an elementary reaction process $\left(\mathrm{F}+\mathrm{H}_{2}\right)$ was studied [14]. A very strong dependence of the new reaction rate coefficient on powerlaw parameter was shown. Then the tunneling correction was considered in generalized transition state theory and applied into $\mathrm{H}+\mathrm{H}_{2}$ reaction [17]. There are only minor differences arising due to the q-parameter between $k_{\mathrm{q}}$ and $k$.

The pre-exponential factors of $\mathrm{F}+\mathrm{H}_{2}, \mathrm{CO}+\mathrm{O}_{2}$, and $\mathrm{CH}_{3}+\mathrm{CH}_{3}$ were calculated according to generalized collision theory [21]. The power-law rate coefficient in collision theory also strongly depends on the power-law parameter. Consequently, the new collision theory can overcome the difficulties in the Lindemann-Christiansen mechanism. Four barrierless reactions were taken as application examples with larger fitting $q$-parameters can be exactly in agreement with measurements in the experimental studies [25]. Unlike those for bimolecular and unimolcular reactions, due to the lack of barriers, the power-law rate coefficient for barrierless reactions does not have a power-law function, and thus is not very strongly dependent on the $q$-parameter.

Two unimolecular reactions, the dissociation of $\mathrm{CH} 3 \mathrm{CO}$ and the isomerization of $\mathrm{CH} 3 \mathrm{NC}$, were taken as application examples to calculate their reaction rates [26]. It should be mentioned that the famous RRKM (after Rice, Ramsperger, Kassel, and Marcus) theory is in fact the high pressure limit of unimolecular reaction theory.

In a word, with different fitting $q$-parameters, all the rate coefficients of chemical reactions above can be calculated exactly in agreement with measurements in the experimental studies in the studied temperature ranges. 


\section{Conclusion}

First, the generalized transition state theory for an elementary bimolecular reaction was studied based on the power-law nonextensive statistical mechanics theory. A generalized reaction rate formula with power-law distribution was obtained, which shows that the reaction rate not only depends on a power-law parameter but on the reaction coordinate frequency in the transition state. The example of $\mathrm{F}+\mathrm{H}_{2}$ was taken and the new theory shows a better agreement with all the experimental results. What's more, the tunneling correction was considered into generalized transition state theory mentioned above, which could be very important for the hydrogen reactions in low temperature. Similar results were given for the tunneling reaction.

Second, the collision theory was further generalized and studied. The reaction rate formula was obtained according to the assumption that the system follows the power-law distribution. The generalized collision theory can effectively overcomes two important difficulties of the famous Lindemann mechanism. The pre-exponential factors of $\mathrm{F}+$ $\mathrm{H}_{2}, \mathrm{CO}+\mathrm{O}_{2}$, and $\mathrm{CH}_{3}+\mathrm{CH}_{3}$ were calculated. In addition, the reaction without barrier with power-law distribution was studied in the framework of Tsallis statistics.

Last but not least, the generalized unimolecular reaction theory rate was considered and discussed. The two reaction rate formulae in high pressure limit and low pressure limit were rigorously given respectively with power-law distributions. The decomposition of $\mathrm{CH}_{3} \mathrm{CO}$ and the isomerization of $\mathrm{CH}_{3} \mathrm{NC}$ were taken as two examples, all of which could be well described by generalized theoretical results. In sum, a great deal of work have been done to develop models of phenomenological kinetics with non-Maxwell-Boltzmann distributions and it is expected that the generalized theories may give a bright direction in the near further.

\section{Reference}

1. Lenzi, E. K., Anteneodo, C., Borland, L., Phys. Rev. E., 2001, 63, 051109-1.

2. Zhao, J. L., Bao, J. D., Commun. Theor. Phys., 2005, 44, 752.

3. Aquilanti, V., Mundim, K. C., Elango, M., Kleijn, S., Kasai, T., Chem. Phys. Lett., 2010, 498, 209.

4. Silva, V. H. C., Aquilanti, V., de Oliveira, H. C. B., Mundim, K.
C., Chem. Phys. Lett., 2013, 590, 201.

5. Frauenfelder, H., Sligar, S. G., Wolynes, P. G., Science, 1991, 254, 1598.

6. Skokov, V. N., Reshetnikov, A. V., Koverda, V. P., Vinogradov, A. V., Physica A, 2001, 293, 1.

7. Furusawa, C., Kaneko, K., Phys. Rev. Lett., 2003, 90, 088102.

8. Benz, R. W., Swamidass, S. J., Baldi, P., J. Chem. Inf. Model, 2008, 48, 1138.

9. Kaneko, K., Orig. Life Evol. Biosphys., 2007, 37, 449.

10. Nacher, J. C., Akutsu, T., Cell. Biochem. Biophys., 2007, 49, 37.

11. Tsallis, C., J. Stat. Phys., 1988, 52, 479.

12. Tsallis, C., Introduction to Nonextensive Statistical Mechanics: Approaching a Complex World, New York: Springer, 2009.

13. Pollak, E., Talkner, P., Chaos, 2005, 15, 026116.

14. Yin, C. T., Du, J. L., Physica A, 2014, 395, 416.

15. Hanggi, P., Talkner, P., Borkovec, M., Rev. Modern Phys., 1990, $62,251$.

16. Hu, Y., Lv, R., Liu, G., Hei, E., Physical Chemistry, 5th ed., Higher Education Press, Beijing, 2007.

17. Yin, C. T., Zhou, Y. J., Du, J. L., Physica A, 2014, 413, 294.

18. Bell, R. P., The Tunnel Effect in Chemistry, Chapman \& Hall, London, New York, 1980.

19. Henriksen, N. E., Hansen, F. Y., Theories of Molecular Reaction Dynamics: The Microscopic Foundation of Chemical Kinetics, Oxford University Press, New York, 2008.

20. Arnaut, L., Formosinho, S., Burrows, H., Chemical Kinetics: From Molecular Structure to Chemical Reactivity, Elsevier, 2007.

21. Yin, C. T., Du, J. L., Physica A, 2014, 407, 119.

22. Silva, R., Plastino, A.R., Lima, J.A.S., Phys. Lett. A, 1998, 249, 401.

23. Truhlar, D. G., Garrett, B. C., Klippenstein, S. J., J. Phys. Chem., 1996, 100, 12771.

24. Fernandez-Ramos, A., Miller, J. A., Klippenstein, S. J., Truhlar, D. G., Chem. Rev., 2006, 1064518.

25. Yin, C. T., Du, J. L., J. Stat. Mech. 2014, P07012.

26. Yin, C. T., Guo, R., Du, J. L., Physica A, 2014, 408, 85.

27. Mathai, A. M., Haubold, H. J., Special Functions for Applied Scientists, Springer, New York, 2008.

28. , N. J. B., Comprehensive Chemical Kinetics, Elsevier, 2003.

\section{Cangtao Yin*}

Instituto de Química, Universidade de Brasília, Brasília, Brazil. *Email: ctyin1986@gmail.com 